This document must be cited according to its final version which is published in a journal as:

N. Daraoui ${ }^{1}$, P. Dufour ${ }^{1}$, H. Hammouri ${ }^{1}$, A. Hottot ${ }^{1}$,

"Model predictive control during

the primary drying stage of lyophilisation",

Control Engineering Practice, ISSN: 0967-0661

2010, 18(5), 483-494.

http://dx.doi.org/10.1016/j.conengprac.2010.01.005

All open archive documents of Pascal Dufour are available at: http://hal.archives-ouvertes.fr/DUFOUR-PASCAL-C-3926-2008

The professional web page ( $\mathrm{Fr} / \mathrm{En}$ ) of Pascal Dufour is:

http://www.lagep.univ-lyon1.fr/signatures/dufour.pascal

The professional web page ( $\mathrm{Fr} / \mathrm{En}$ ) of Hassan Hammouri is: http://www.lagep.univ-lyon1.fr/signatures/hammouri.hassan

The web page of this research group is:

http://hal.archives-ouvertes.fr/SNLEP

1

Université de Lyon, Lyon, F-69003, France; Université Lyon 1;

CNRS UMR 5007 LAGEP (Laboratoire d'Automatique et de GEnie des Procédés),

43 bd du 11 novembre, 69100 Villeurbanne, France

Tel +33 (0) 472431845 - Fax +33 (0) 472431699

http://www-lagep.univ-lyon1.fr/ http://www.univ-lyon1.fr http://www.cnrs.fr 


\title{
Model predictive control
}

\section{during the primary drying stage of lyophilisation}

\author{
N. Daraoui ${ }^{1}$, P. Dufour ${ }^{2}$, H. Hammouri and A. Hottot \\ Université de Lyon, F-69622, France; \\ Université Lyon 1, Villeurbanne; \\ CNRS, UMR 5007, LAGEP;
}

43 bd du 11 novembre, 69622 Villeurbanne Cedex, France

During the primary drying stage of pharmaceutical solutions in vial, the sublimation front is the boundary between the dried and frozen layers that moves from the top of the vial to its bottom. While only few on-line measures are available, it is an important variable to control. This paper deals with the on-line partial differential equation model-based predictive control of the sublimation front position, assuming two strategies based on various availability of measurement used in the feedback loop. Through the MPC@CB control software, the robustness of the controller with respect to the main model parameter uncertainty is shown.

Keywords: Process control, model predictive control, chemical engineering, lyophilisation, drying, heat transfer, mass transfer.

\footnotetext{
${ }^{1}$ The authors acknowledge the AUF (Agence Universitaire de la Francophonie) for funding this postdoctoral position.

${ }^{2}$ Corresponding author: dufour@lagep.univ-lyon1.fr. Fax: +33 (0) 4724316 99. tinyurl.com/dufourpascal
} 


\section{Introduction}

Freeze drying, also called lyophilisation, is a drying process used in biotechnology, food and pharmaceutical industries frequently used to stabilize and preserve products (Pikal, 2006). Compared with conventional drying techniques, freeze drying is generally considered to produce the dried product with the highest quality. Until now, a larger use of the freeze drying is limited by its cost and its use remains therefore limited for high value added products, like pharmaceutical products. The main reason for the high cost of freeze drying is the need to operate under a vacuum, which involves an important treatment time. Major costs are due to: the energy for the sublimation of frozen solvent during the primary drying stage, the energy to maintain the vacuum and overhead costs which are all functions of the drying time and the energy for the removal (desorption) of bound (unfrozen) solvent during the primary and secondary drying stages. The sublimation front is the moving boundary between the dried and frozen layers that moves until it reaches the bottom of the sample, hence describing the end of the primary drying stage. At the end of the primary drying stage, the control objectives change to start the secondary drying stage. The control of the sublimation front is therefore important. Moreover, one very important parameter in the study of the freeze drying is the temperature of the product which must be carefully controlled during the primary and the secondary drying stages of freeze drying. In (Liapis, Pikal and Bruttini, 1996), the future research needs in freeze drying are identified which emphasis on: pharmaceuticals and biotechnology products formulation, sensing process control during freezing, primary drying stage and secondary drying stage. A primary impediment is that usually very few measurements are available for implementing feedback loop control of such processes. In drying, the issue is that few studies are dealing with process control: this point is summarized in a review paper (Dufour, 2006). Previous studies on the control of the freeze drying process 
obtained optimal policies offline as a trade off between the productivity of the process and the quality of the product. However, these studies assumed a perfect model with model parameters known perfectly. The contribution of this paper is to provide two implementable model-based control strategies to control the sublimation front position (and hence the duration of drying) on-line during the primary drying stage of the freeze drying process of a solution in vial, in presence of constraints and modeling uncertainties.

To satisfy this objective, the development of a mathematical model is preferable, since it may be used with model-based control strategy to predict the behavior of the freeze drying process. Consequently, a number of mathematical freeze drying models have been published in the literature, mainly in the past twenty years. Generally, dynamic models for freeze-drying predict drying behaviors more accurately than steady state models. These take the form of complex transport equations based on partial differential equations (PDEs), which are difficult to solve online and also need variables and parameters that are difficult to measure accurately experimentally.

Model predictive control (MPC) is one of the most popular finite dimensional controllers (Qin and Badgwell, 2003). Thousands of industrial applications of MPC exist today, particularly in the chemical and petrochemical industries. The idea of MPC is to solve an on-line open-loop model-based optimization problem over a finite prediction horizon in order to find the value of the manipulated variable that has to be implemented. The procedure is repeated at each sample time with the model being updated with process measurements. The first main advantage is that constraints, due to manipulated variables physical limitations, operating procedures or safety reasons, etc., may be explicitly specified into the problem formulation. The second main advantage of MPC is its ability to address long time delays, inverse responses, significant non-linearities and multivariable interactions. However, a numerical technique must be used to solve this optimal problem, where the on-line computational effort 
may become a limiting factor in their implementation. Therefore, significant effort is being devoted towards development of fast MPC techniques (Diehl et al., 2002; Schäfer, Kühl, Diehl, Schlöder and Bock, 2007; Mahmood and Mhaskar, 2008; Zavala and Biegler, 2009) even for large-scale systems. The method used here (Dufour, Touré, Blanc, and Laurent, 2003) is developed for problems where the time needed to compute the solution of the model (which is based on PDEs) may be important (with respect to the relatively short sampling time) and where few iterations are allowed for the optimizer to give a solution. Therefore, the main idea of this method is that the model is solved rapidly and the input (hard) constraints are satisfied at any time, in order to be physically applicable. Moreover, due to the short time available, since the output (soft) constraints may not all be satisfied at any time, the smallest constraint violation can be found. Before any future experimental implementation of the proposed approach, the first step is therefore to evaluate, with simulations, the closed loop performance of the MPC controller and is the focus of this paper.

This paper is organized as follows: First, the non-linear parabolic PDE model describing the primary drying stage of the freeze drying process is presented. Then the general framework of the model predictive control approach is developed. Finally, this MPC approach is evaluated for the control of the freeze drying process. In order to control the sublimation front position, two closed loop control problems are presented here, assuming various availability of measurement used in the feedback loop: First, a temperature trajectory tracking (measured at the bottom of the sample) is used for inferential control (Doyle III, 1998) of the sublimation front position. Secondly, the maximization of the constrained sublimation front velocity is implemented assuming the availability of the on-line measure of the sublimation front position. The simulations are implemented using the MPC@CB software.

\section{Freeze drying process modeling}


Freeze-drying is a drying process where the solution is first frozen at low temperature, thereby converting most of the water into ice. Unfrozen water remains in the freeze concentrated phase which must be maintained under its glass transition temperature $T_{g}$. The ice is then removed by sublimation at low temperature and at low pressure during the primary drying stage of the process. This gives an opportunity to avoid denaturation of the product caused by heating at low temperature. In the pharmaceutical industry, the solution is normally filled into glass vials which are placed on temperature controlled shelves in a large vacuum chamber. The shelf temperature is lowered to freeze the product. After complete solidification, the pressure in the chamber is lowered to initiate rapid sublimation. Sublimation begins at the top of the material and creates the interface between the frozen and dried layers. This interface moves through the material until only a dried porous material remains at the end of the primary drying stage. In general, the upper surface heat input of the dried layer is supplied by radiation from the top of the sample through the gas phase. This heat is transferred by conduction to the sublimation interface through the porous dried layer. The bottom surface heat input of the frozen layer is supplied by the heating plate and is transferred by conduction to the sublimation interface through the frozen layer of the sample. Freeze drying involves simultaneous heat and mass transfers which have to be taken into account when models are developed.

\subsection{Existing models}

Models for prediction of freeze-drying are based on complex transport equations, which are difficult to solve and based on variables and parameters difficult to measure accurately experimentally. A number of freeze-drying models have been published in the literature, first 
as steady state models (like the uniformly retreating ice front (URIF) model of (King, 1971)), and later featuring dynamics characteristics. A brief summary of these models follows:

- In (Liapis and Litchfield, 1979a), one dimensional heat and mass transfer was assumed, the temperature and pressure profiles inside the dried and frozen regions were modeled using PDEs, which in turn, allowed modeling of the sublimation front position.

- In (Litchfield and Liapis, 1979b), a PDE model was based on material and energy balances in the dried and frozen regions of a material under freeze drying. Both sublimation and adsorption of unfrozen water were accounted for.

- In (Liapis and Bruttini, 1994), a PDE model-based theory was given to describe quantitatively the dynamic behavior of the primary and secondary drying stages of the freeze-drying of pharmaceutical crystalline and amorphous solutes.

- According to the previous PDE model of (Liapis and Bruttini, 1994), (Sadikoglu and Liapis, 1997) showed that the contribution of removal of bound water was not significant for skim milk.

- In (Mascarenhas, Akay and Pikal, 1997), both the primary and secondary drying stages were modeled in 2 spatial dimensions using a finite element formulation. The time-wise variation and spatial profiles of the partial pressure of water vapor, the temperature, the concentration of sorbed water and the position of the sublimation front were modeled.

- (Delgado and Sun, 2001) presented a review on freeze drying, where it was underlined that heat transfer coefficients were hard to estimate with typical uncertainties of $\pm 20 \%$ and that data on mass transfer coefficients were scarce.

- (Brülls and Rasmuson, 2002) showed an experimental and theoretical heat transfer study on the freezing of liquid solutions in commercial glass vials for industrial operating conditions. A dynamic two spatial dimension PDE model based on Fourier's law allowed 
investigating the effects of different factors (vial type and shape, curvature of the vial bottom, total chamber pressure, filling height, and vial position on the shelf).

- In (Boss, Filho and Vasco de Toledo, 2004), the proposed mathematical model better described the real data compared to the model of (Sadikoglu and Liapis, 1997). Influence of the value of certain model parameter over freeze drying was shown.

- In (Hottot, Peczalski, Vessot and Andrieu, 2006a), a 2 spatial dimension PDE model simulated the temperature profiles and the sublimation front velocities during the sublimation step of the freeze drying of Bovin Serum Albumin (BSA)-based formulations for typical industrial freeze-drying conditions. This finite element code was also used in (Nakagawa, Hottot, Vessot and Andrieu, 2007) to estimate the ice crystal size distribution.

- In (Sane and Hsu, 2008), a coupled PDE and ordinary differential equation (ODE) model predicted the product temperature profiles during primary and secondary drying stages, and chamber pressure, condenser temperature and nitrogen flow rate.

- In (Liu, Zhao and Feng, 2008), a set of exergy analysis model was established to investigate exergy losses in a freeze-drying cycle.

The works of Liapis'team in the late seventies was therefore the beginning of the modeling in lyophilisation and it influenced the next generations of model.

\subsection{Mathematic model}

To carry out the objective of this paper, a one spatial dimension freeze drying model based on the work of (Sadikoglu and Liapis, 1997) for a standard simplified formulation is considered. BSA is used because of its stability, its lack of effect in many biochemical reactions, and its low cost since large quantities may be obtained. The freeze drying process during the primary drying stage is illustrated in Figure 1. During the primary drying stage, the product is 
composed of two phases: a dry layer (index 1 in the model), in which the majority of water is sublimated and a frozen layer (index 2 in the model). These two areas are separated by a moving interface called the sublimation ice front described by the space coordinate $z$ at $z=H(t)$. The quantities $q_{1}$ and $q_{2}$ shown in Figure 1 represent the heat flux at the top and the bottom of the vial respectively, including the effects of radiation and conduction.

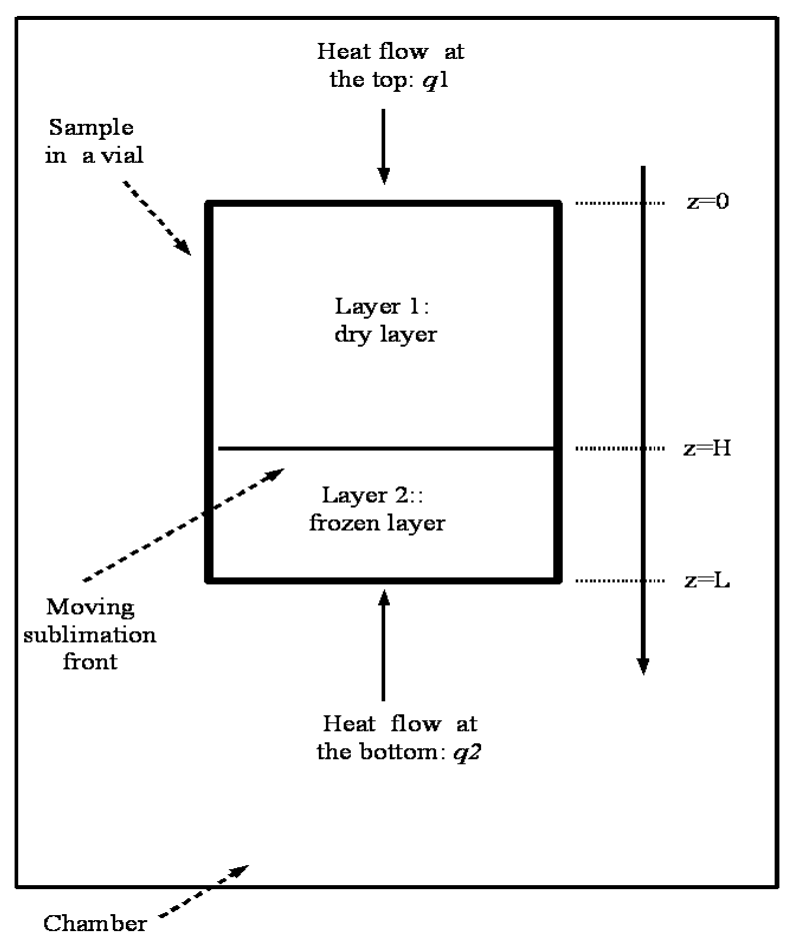

Figure 1: A schematic of the freeze drying process during the primary drying stage.

In order to decrease the on-line computational load for the model-based control algorithm, it is assumed:

- One dimensional heat and mass transfers across $z$.

- The sublimation front is planar and parallel to the horizontal section of the vial. 
- The gas phase inside the pores of the dry layer is only composed of pure water vapor: the effect of inert gas is negligible because the amount of inert gas in the drying chamber is much smaller than the water vapor.

- The value of the partial pressure of water vapor at the top of the dry layer is equal to the total pressure in the sublimation chamber.

- The frozen region is considered to be homogeneous with uniform thermal conductivity, density and specific heat.

- Desorption of the sorbed water in the dried layer may occur during the primary drying stage.

- Side wall heat flux is negligible.

Based on these assumptions, the mathematical model consists of the unsteady state energy balance in the dried and frozen regions:

$$
\left\{\begin{array}{l}
\frac{\partial T_{1}(z, t)}{\partial t}=\frac{k_{1 e}\left(T_{1}(H, t), t\right)}{\rho_{l e^{c} p 1}} \frac{\partial^{2} T_{1}(z, t)}{\partial z^{2}}-\frac{c_{p g}}{\rho_{l e} c_{p 1}} \frac{\partial\left(N_{w}\left(T_{1}(H, t), H(t)\right) T_{1}(z, t)\right)}{\partial z} \ldots \\
\quad \ldots .-\frac{\Delta H_{v} \rho_{1}}{\rho_{1 e} c_{p 1}} k_{d} C_{s w}(z, t), 0<z<H(t), t>0 \\
\frac{\partial T_{2}(z, t)}{\partial t}=\frac{k_{2}}{\rho_{2} c_{p 2}} \frac{\partial^{2} T_{2}(z, t)}{\partial z^{2}}, H(t)<z<L, t>0 \\
\frac{d H(t)}{d t}=-\frac{1}{\rho_{2}-\rho_{1}} N_{w}\left(T_{1}(H, t), H(t)\right), t>0 \\
\frac{\partial C_{s w}(z, t)}{\partial t}=-k_{d} C_{s w}(z, t), 0 \leq z \leq H(t), t>0
\end{array}\right.
$$

where the two independent variables are the time $t$ and the space $z$. $L$ is the length of the sample. The state variable $x(z, t)$ is constituted by: the dried layer temperature $T_{l}(z, t)$, the 
frozen layer temperature $T_{2}(z, t)$, the sublimation front position $H(t)$, and the concentration of bound water $C_{s w}(t)$. The model parameters which do not depend on $t$ or $\boldsymbol{z}$ are constant values. The value used for the model parameters may be found in (Hottot, Daoussi and Andrieu, 2006b) and are summarized in the nomenclature with the model variables.

In order to specify the remaining functions in this model, let us first define $p_{0}$ and $p_{H}\left(T_{l}(H, t)\right)$, the partial pressures of water vapor at $z=0$ and $z=H(t)$ respectively. The pressure boundary condition at the top surface of the material being dried $\left(p_{0}\right)$ is defined as a constant pressure inside the drying chamber, and the vapor pressure at the sublimation interface $\left(p_{H}\left(T_{1}(H, t)\right)\right)$ is defined as an equilibrium vapor pressure according to the temperature at the moving interface. The pressure $p_{H}\left(T_{1}(H, t)\right)$ is given by (Chouvenc, Vessot, Andrieu and Vacus, 2004):

$$
\left\{\begin{array}{l}
p_{H}\left(T_{1}(H(t), t)\right)=p_{H \infty} \exp \left(-\frac{A}{T_{1}(H(t), t)}\right), t>0 \\
p_{H \infty}=\exp (B)
\end{array}\right.
$$

Based on the diffusion equations by Evans, a simplified version of the water vapor mass flux $N_{w}\left(T_{1}(H, t), H(t)\right)$, used in the model (1.) and defined in (Liapis and Litchfield, 1979a), is:

$$
N_{w}\left(T_{1}(H, t), H(t)\right)=-\frac{M_{w} k_{1}}{R_{i g} T_{1}(H(t), t) H(t)}\left(p_{H}\left(T_{1}(H(t), t)\right)-p_{0}\right), t>0
$$

The partial pressure of water vapor at $z=0\left(p_{0}\right)$ may also be used as a manipulated variable. Here a constant value is assigned to this pressure $\left(10 \mathrm{~N} . \mathrm{m}^{-2}\right)$. The thermal conductivity in the dried layer $k_{l e}\left(T_{1}(H, t)\right)$ is given by the following expression (Sheehan and Liapis, 1998): 


$$
k_{1 e}\left(T_{1}(H(t), t)\right)=k_{1 e 1} *\left(p_{0}+p_{H}\left(T_{1}(H(t), t)\right)\right)+k_{1 e 2}, t>0
$$

The initial conditions for the equations in the dynamic system (1.) are given by:

$$
\begin{cases}T_{1}(z, t)=T^{o} & , 0 \leq z \leq H(t), t=0 \\ T_{2}(z, t)=T^{o} & , H(t) \leq z \leq L, t=0 \\ H(t)=0^{+} & , t=0 \\ C_{s w}(z, t)=C_{s w}^{0} & , 0 \leq z \leq L, t=0\end{cases}
$$

The boundary conditions for the system (1.-4.) are as follows:

$$
\begin{cases}-k_{1 e}\left(T_{1}(H(t), t)\right) \frac{\partial T_{1}(z, t)}{\partial z}=q_{1}(t)=\sigma F_{u p}\left(T_{u p}^{4}(t)-T_{1}^{4}(0, t)\right), z=0, t>0 & \\ k_{2} \frac{\partial T_{2}(z, t)}{\partial z}-k_{1 e}\left(T_{1}(z, t)\right) \frac{\partial T_{1}(z, t)}{\partial z}=\ldots & , z=H(t), t>0 \\ \left(-\Delta H_{S}-\frac{\rho_{2} c_{p 2} T_{2}(z, t)-\rho_{1} c_{p 1} T_{1}(z, t)}{\rho_{2}-\rho_{1}}\right) N_{w}\left(T_{1}(z, t), H(t)\right) & , z=H(t), t>0 \\ T_{1}(z, t)=T_{2}(z, t) & , z=L, t>0 \\ k_{2} \frac{\partial T_{2}(z, t)}{\partial z}=q_{2}(t)=-h_{v}\left(T_{l p}(t)-T_{2}(L, t)\right) & \end{cases}
$$

where $T_{u p}(t)$ and $T_{l p}(t)$ are the temperatures of the lower and upper heating plates respectively, that are used in this study as manipulated variables.

\section{Assumption 1:}

The temperature of the upper and lower heating plates are the same, i.e. $T_{u p}(t)=T_{l p}(t)$. 
Based on assumption 1, the manipulated variable $u$ is $T_{u p}$ and the state variable $x$ is $\left(\begin{array}{llll}T_{1} & T_{2} & H & C_{S w}\end{array}\right)^{T}$.

Freeze drying constitutes, therefore, a typical moving boundary problem: the motion of the sublimation interface is essential for accurate prediction of the freeze drying characteristics. In order to be used in the model-based control software, the equations of the model must first be expressed in a new coordinates system (Duda, Malone, Notter and Vrentas, 1975):

$$
\begin{cases}\xi_{1}=\frac{z}{H(t)} & , 0<z \leq H(t), t \geq 0 \\ \xi_{2}=\frac{H(t)-z}{H(t)-L} & , H(t) \leq z \leq L, t>0\end{cases}
$$

Let us define new state variables as follows:

$$
\left\{\begin{array}{l}
\tilde{T}_{1}\left(\xi_{1}, t\right)=T_{1}(z, t) \\
\tilde{T}_{2}\left(\zeta_{2}, t\right)=T_{2}(z, t) \\
\tilde{C}_{s w}\left(\xi_{1}, t\right)=C_{s w}(z, t)
\end{array}\right.
$$

Therefore, the dynamic equations of the initial model (1.-4.) can be written as follows: 


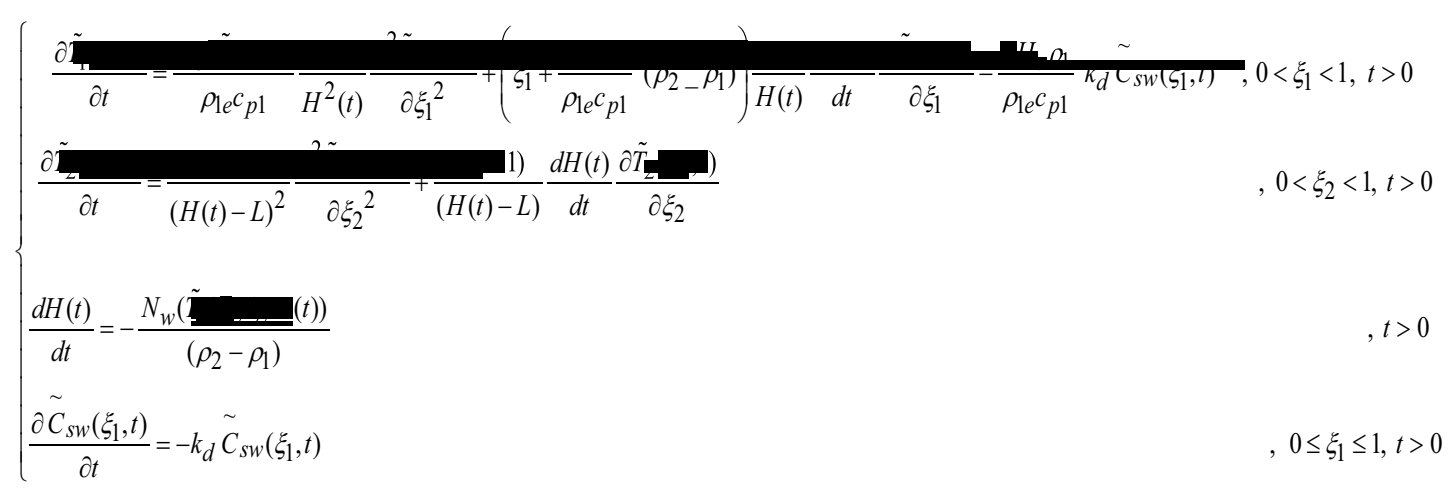

The initial conditions (5.) become:

$$
\left\{\begin{array}{lll}
\tilde{T}_{1}\left(\xi_{1}, t\right)=T^{o} & , 0 \leq \xi_{1} \leq 1, & t=0 \\
\tilde{T}_{2}\left(\xi_{2}, t\right)=T^{o} & , 0 \leq \xi_{2} \leq 1, & t=0 \\
H(t)=0^{+} & , t=0 \\
\tilde{C}_{s w}\left(\xi_{1}, t\right)=C_{s w}^{0} & , 0 \leq \xi_{1} \leq 1, & t=0
\end{array}\right.
$$

The boundary conditions (6.) become:

$$
\left\{\begin{array}{lr}
-\frac{k_{1 e}\left(\tilde{T}_{1}\left(\xi_{1}, t\right)\right.}{H(t)} \frac{\partial \tilde{T}_{1}\left(\xi_{1}, t\right)}{\partial \xi_{1}}=\sigma F_{u p}\left(T_{u p}^{4}(t)-\tilde{T}_{1}^{4}(0, t)\right) & , \xi_{1}=0, t>0 \\
\frac{k_{2}}{(H(t)-L)} \frac{\partial \tilde{T}_{2}\left(\xi_{2}, t\right)}{\partial \xi_{2}}-\frac{k_{1 e}\left(\tilde{T}_{1}\left(\xi_{1}, t\right)\right.}{H(t)} \frac{\partial \tilde{T}_{1}\left(\xi_{1}, t\right)}{\partial \xi_{1}}=\left(-\Delta H_{s}-\frac{\rho_{2} c_{p 2} \tilde{T}_{2}\left(\xi_{2}, t\right)-\rho_{1} c_{p 1} \tilde{T}_{1}\left(\xi_{1}, t\right)}{\rho_{2}-\rho_{1}}\right) N_{w}\left(\tilde{T}_{1}\left(\xi_{1}, t\right), H(t)\right), \xi_{1}=1, \xi_{2}=0, t>0 & , \xi_{1}=1, \xi_{2}=0, t>0 \\
\tilde{T}_{1}\left(\xi_{1}, t\right)=\tilde{T}_{2}\left(\xi_{2}, t\right) & , \xi_{2}=1, t>0 \\
-\frac{k_{2}}{(H(t)-L)} \frac{\partial \tilde{T}_{2}\left(\xi_{2}, t\right)}{\partial \xi_{2}}=-h_{v}\left(T_{l p}(t)-\tilde{T}_{2}(L, t)\right) &
\end{array}\right.
$$

One can clearly note that the model (9.-11.) represents a strong non-linear formulation described by a one-dimensional PDE model. Also, the effect of the moving boundary on the dynamics of both the temperatures is clearly underlined since a non-linear convective term 
now appears. This model belongs to a more general class of one dimensional non-linear parabolic PDE based model with boundary control, whose class is summarized as follows:

$$
\left(S_{N L}\right) \begin{cases}\frac{\partial x_{m}(\zeta, t)}{\partial t}=F_{d}\left(x_{m}(\zeta, t)\right), & , \forall \zeta \in \Omega, t>0 \\ F_{b}\left(x_{m}(\zeta, t), u(t)\right)=0 & , \forall \zeta \in \partial \Omega, t>0 \\ x_{m}(\zeta, 0)=x_{m}^{0} & , \forall \zeta \in \Omega \cup \partial \Omega, t=0 \\ y_{m}(t)=C x_{m}(\zeta, t) & , \forall \zeta \in \Omega \cup \partial \Omega, t>0\end{cases}
$$

where $\zeta$ is the independent space variable, $\Omega$ is the spatial domain, $\partial \Omega$ is its boundary and $t$ is the independent time variable. $x_{m}$ is the model state belonging to an adequate infinite dimensional state space, $u$ is the control signal (or manipulated variable), $y_{m}$ is the model output, $F_{d}$ and $F_{b}$ are non-linear operators and $C$ is a linear operator.

\section{Assumption 2:}

The solution of $\left(S_{N L}\right)$ is unique and depends continuously on its initial value.

In terms of parameter uncertainty, (Hottot et al., 2006b) showed that the heat capacity of the frozen layer $C_{p 2}$ was not known accurately and changed according to the manipulated temperature variable: between $240 \mathrm{~K}$ and $260 \mathrm{~K}$, it varied up to $50 \%$ around a nominal value. Moreover, it had a large impact over the state variable dynamics. This motivates the use of a closed loop controller for such process, which is discussed in the section 4.

\section{Freeze drying process control strategy}

\subsection{Existing control approaches}


In terms of tuning of the control variables, suitable shelf temperature and chamber pressure conditions are frequently established empirically off-line in a "trial - and - error" experimental approach or by factorial design. Some freeze-drying control studies were published:

- (Meo III and Friedly, 1973) seems to be the first work dealing with the control of freeze dryer. The control approach was not discussed but allows decreasing the drying time.

- In (Liapis and Litchfield, 1979a), a quasi steady state model was used in a lagrangian approximation to decrease the drying time. The control policies consisted of 4 segments where each of the 2 manipulated variables were constant and were tuned off-line to prevent the surface and interface temperatures from exceeding their maximum allowable. This was later extended to the dynamic model case in (Litchfield and Liapis, 1982).

- In (Lombraña and Díaz, 1987a), a quasi-steady PDE model was used, where the mass transfer coefficient in the dried region was assumed to vary according to experimental knowledge. The control variable was the plate temperature and was tuned according to a programmed heating strategy which accounted for the constraints on the temperature. The control law was not detailed. Compared with the constant heating strategy, this strategy allowed decreasing the drying time. In the multivariable control case (adding the pressure as control variable), the drying time was shorter (Lombraña and Díaz, 1987b).

- In (Lombraña and Villarán, 1997), the optimal open loop temperature and pressure profiles in the fluid bed were obtained by a short factorial design.

- In (Sadikoglu, Liapis and Crosser, 1998), a lagrangian method was used to find the optimal control of the primary and secondary drying stages, assuming a perfect PDE model. In (Sadikoglu, Ozdemir and Seker, 2003), this approach was used with a two spatial dimension PDE model for the optimal control of the primary drying stage.

- In (Boss, Filho and Vasco de Toledo, 2004), compared to a drying with constant control values, an open loop model-based optimization strategy (using sequential quadratic 
programming) allowed to decrease the primary and secondary drying times by $1.5 \mathrm{~h}$, while removing the same amount of bound water.

- In (Tang, Nail and Pikal, 2005), for primary and secondary drying stages, a procedure to optimize the freeze-drying process was developed by combination of feedback information from temperature measurement during lyophilisation, expert system and steady state heat and mass transfer theory.

- In (Barresi et al., 2009), a software was developed for determining the optimal shelf temperature for primary drying of the lyophilisation process of pharmaceuticals in vials, ensuring the fastest drying time without overcoming the maximum allowable product temperature both in scouting and production cycles. It was based on a relatively simple model.

- In (Fissore, Velardi and Barresi, 2008), 2 approaches were discussed. Firstly, an ideal model-based control strategy continuously changed the shelf temperature in order to maintain the product temperature at a safe level, while minimizing the drying time. Then, a more realistic approach was presented in which an adaptive PI controller with optimal on-line tuning was based on the feedback obtained by an observer of the maximum product temperature.

Recently, the $\mathrm{PhD}$ thesis work of Velardi (Velardi, 2004) dealt with mathematical modeling, observer design and control in lyophilisation. These models (Velardi and Barresi, 2008) could be used for off-line optimization of the freeze drying cycle, as well as for on-line purposes such as process monitoring. This led to the design of a PI controller with adaptive, modelbased tuning of the P and I modes that also utilized observer techniques for the feedback data (Velardi, Hammouri and Barresi, 2005).

Another issue in lyophilisation deals with the lack of in-situ sensors required by a closed loop controller: indeed, it is often not possible to have a physical sensor embedded in the sample. 
Most of the time, only pressure and temperature measurements are available at certain points. Therefore, some recent works are focusing on the development of sensors. (Hottot, Andrieu, Shalaev, Gatlin and Ricketts, 2009a; Hottot et al., 2009b) compared the use of physical sensors (hygrometer, pressure gauge, thermocouples, etc.) on a syringe rack. Another idea was use of model-based sensors (Besançon, 2007), also known as observers. (Velardi et al., 2005; Velardi, Hammouri and Barresi, 2009) and (Barresi et al., 2008) dealt with observer based techniques that allowed estimating temperature and interface position online.

In this work, the use of a closed loop model-based control approach is advocated, since it allows handling model parameter uncertainties. A PDE model is used in a predictive controller to tune online the shelve temperatures according to the control objective and the various measurements that are assumed available.

\subsection{Control of PDE systems}

The majority of control applications focus on processes whose dynamic behavior may be modeled by relatively simple models usually consisting of ODEs. However, most chemical systems, like the freeze drying process, are characterized by non-linear distributed parameter systems which are naturally modeled by non-linear PDEs. In control theory, due to the complexity of the problem, relatively few studies are devoted to the control of processes explicitly characterized by PDE models, especially in the non-linear case. The more popular way to synthesize finite dimensional controllers for infinite dimensional systems is to first construct a finite approximation of the model which serves as the foundation for the finite dimensional controller. Even if various finite dimensional methods are proposed to control such distributed parameter systems, there is no general framework yet. The original PDE model is usually simplified into an ODE model based on: finite differences method, finite 
volume method, orthogonal collocation method, Galerkin's method, or on modal decomposition. Many works exist with these approximation techniques. Recently (Dubljevic, Mhaskar, El-Farra, Christofides, 2005) used the Galerkin's method to capture the dominant dynamics of the (PDE based) transport-reaction processes in order to design a MPC. In (Dubljevic and Christofides, 2006), a modal decomposition technique was used to decompose the system into a finite dimensional (slow) subsystem coupled with an infinite dimensional (fast) subsystem. Various state feedback predictive controllers were then designed. In (Damak, 2007), the design of an asymptotic estimator of state and time-varying parameters in the case of a non-linear distributed parameter bioreactor was presented. The structure of the estimator was based on an approximated model of the bioreactor behavior using orthogonal collocation. In (Ravindran, 2007), the optimal boundary feedback stabilization of NavierStokes equations using model reduction was presented. The model reduction was carried out using a combination of proper orthogonal decomposition (POD) and Galerkin projection, and used for the optimal linear quadratic regulator (LQR) synthesis. In (Li and Christofides, 2008), two computationally efficient approaches were presented for the optimal control of diffusion-convection reaction processes described by parabolic PDEs subject to Danckwerts boundary conditions. It was based on reduced-order models combined with a LQR. In (Christofides, El-Farra, Li and Mhaskar, 2008), an overview of recently developed control methods for PDE based models was presented, with examples on crystallization, aerosol and thermal spray. In (Maidi, Diaf and Corriou, 2009), a PDE model combined with differential geometry has been applied to compare two boundary control strategies for the temperature of the liquid fluid at the outlet of a heat exchanger. In (Padhiyar and Bhartiya, 2009), the control of the spatial property profile was discussed, since the endpoint itself is a manifestation of the reaction path and a particular path adopted may offer advantages over others. Based on a MPC formulation, a lexicographic optimization was used to prioritize the different sections of 
the profile when the target profile specified was unachievable. Cascaded continuous stirred tank reactors were used to approximate the model of a pulp digester.

\subsection{Model Predictive Control approach employed}

A particular PDE model-based predictive control strategy detailed in (Dufour et al., 2003) is used here. The idea of this approach is to transform the initial PDE model-based constrained control problem into an ODE model-based unconstrained control problem, such that the time needed to solve the on-line optimization problem is less than the sampling time. Therefore, such a controller may be implemented for the online control of a real plant. The drawback of this approach is that it requires several approximations, both in the resolution method and in the model (which is solved in an open loop sense) by the MPC that may impact the closed loop performance. In the pasta dryer control (De Temmerman, Dufour, Nicolaï and Ramon, 2009), using such a linearized model based strategy instead of a non-linear model based strategy contributed to a significant reduction in the online computational time needed by the MPC (by a factor of 5), without compromising the closed loop performance. In this approach, the initial general optimization problem is formulated into the future over a receding horizon $N_{p}$, where the cost function $J$ aims to reflect any control problem (trajectory tracking, processing time minimization, energy consumption minimization, etc.):

$$
\min _{u} J(u)=\sum_{j=k+1}^{j=k+N_{p}} h_{1}\left(y_{r e f}(j), e(k), y_{m}(j), u(j)\right)
$$

where $k$ is the actual discrete time ( $\left.t=k^{*} T e\right), T e$ is the sampling time, $j$ is the future discrete time index. $y_{p}$ is the process controlled output that has to follow as best as possible the prescribed reference $y_{\text {ref. }}$ The internal model closed loop control structure is used, where the 
control $u$ is applied on both the process and the model, and where the feedback term for the model update is:

$$
e(k)=y_{p}(k)-y_{m}(k), \forall k>0
$$

\section{Assumption 3:}

In order to forecast the process output $y_{p}$ into the future $j$, it is assumed that the error $e(j)=y_{p}(j)-y_{m}(j)$ is constant into the future: $e(j)=e(k)$ is obtained after each update of the measure at time $k$.

$$
y_{p}(j)=y_{m}(j)+e(k), \forall k>0, \forall j \in\left[k+1, k+N_{p}\right]
$$

The $n$ general output constraints $g_{i}$ related to operating conditions, safety, quality, are formulated as inequality constraints on the measured or estimated output (and the input if needed). Based on the assumption 3 and on the internal model control closed loop structure, the $n$ general output constraints $g_{i}$ are reformulated, based on the feedback term $e(k)$ and model response in the future $y_{m}(j)$ obtained with the model:

$$
g_{i}\left(e(k), y_{m}(j), u(j)\right) \leq 0, \forall k>0, \forall j \in\left[k+1, k+N_{p}\right], \forall j \in I^{n}=\{1, \ldots, n\}
$$

These output constraints $g_{i}$ are handled in the optimization problem through the penalty term $J_{e x t}$, based on the exterior penalty method (Fletcher, 1987):

$$
J_{\text {ext }}(u)=\sum_{j=k+1}^{j=k+N_{p}}\left(\sum_{i=1}^{i=n}\left(w_{i} \max ^{2}\left(0, g_{i}\left(e(k), y_{m}(j), u(j)\right)\right)\right)\right)
$$

where $w_{i}$ is an adaptive positive defined weight. The advantage is that such an approach can handle cases where one (or more) of the output constraints may not be satisfied by 
minimizing the constraint violation. The cost function $J$ and the penalty term $J_{\text {ext }}$ are then combined into $J_{\text {tot }}$ :

$$
\min _{u} J_{\text {tot }}(u)=J(u)+J_{\text {ext }}(u)
$$

Concerning the constraints on the manipulated variable $u$ of the process (the constrained optimization argument in the optimization task), they are stated as:

$$
\left\{\begin{array}{l}
u_{\min } \leq u(k) \leq u_{\max }, \forall k>0 \\
\Delta u_{\min } \leq u(k)-u(k-1) \leq \Delta u_{\max }, \forall k>1
\end{array}\right.
$$

In order to decrease the computational load, $u$ is assumed to be a scalar (i.e. a step function over the receding horizon $N_{p}$ ): therefore, the number of arguments of the optimization problem is reduced to one. This constrained optimization argument $u$ is transformed into the unconstrained optimization argument $d$ through a simple hyperbolic transformation of the magnitude and velocity constraints specified for the manipulated variable $u$ (Dufour et al., 2003). This transformation is parameterized at $k$ by the past data $u(k-1)$ and the specified constraint bounds of (19.). The optimizer argument $d$ is finally used in an online penalized unconstrained optimization problem:

$$
\min _{d} J_{t o t}(d)=J\left(h_{1}\left(y_{r e f}(j), e(k), y_{m}(j), d(j)\right)\right)+J_{\text {ext }}\left(g_{i}\left(e(k), y_{m}(j), d(j)\right)\right)
$$

Widely known and used for its robustness and convergence properties, the LevenbergMarquardt's algorithm is used here and the optimization argument is determined iteratively at each sample time $k$ using the process measurement (or estimation), the model predictions and the cost function $J_{\text {tot }}$. From a practical point of view, the next step in this problem is to reduce the computational time needed to solve the optimization problem during the sampling period. This is achieved in several steps. First, the time needed to solve the non-linear model may be 
greatly reduced when it is linearized first in some manner and then the techniques developed for linear systems are employed online subsequently. Based on (Dufour et al., 2003), the nonlinear PDE model $\left(S_{N L}\right)$ is first formulated offline for a particular tuning of $u=u_{0}$, which leads to the linearization trajectories $x_{0}$ and $y_{0}$ described by $\left(S_{0}\right)$. Then, around these trajectories, small variations of the state $\Delta x_{m}$ and small variations of the model output $\Delta y_{m}$ are connected to the small variations of the control actions $\Delta u$ through a time varying linearized PDE model $\left(S_{T V L}\right)$. Finally, the offline non-linear PDE model $\left(S_{0}\right)$ and the online time varying linearized PDE model $\left(S_{T V L}\right)$ replace the initial online non-linear PDE model $\left(S_{N L}\right)$. More technical details may be found in (Dufour et al., 2003). One practical question deals with the choice of the linearization trajectory (hence the tuning of $u_{0}$ ). It may be obtained as the optimal solution of the control problem, assuming a perfect model (Dufour, Touré, Michaud and Dhurjati, 2004). It may also be obtained with a simple tuning of the control actions (constant values for example), by trial-error with simulation, based on the minimization of the cost function (20.). Secondly, in order to be able to calculate $y_{m}$ into the future as required by the cost function (20.), these PDE models are approximated in finite dimension by a discretization technique (finite difference approach). The initial PDE model-based constrained problem is therefore transformed into an ODE model-based unconstrained penalized problem, where the final internal model structure with MPC (IMC-MPC) is shown in figure 2. 


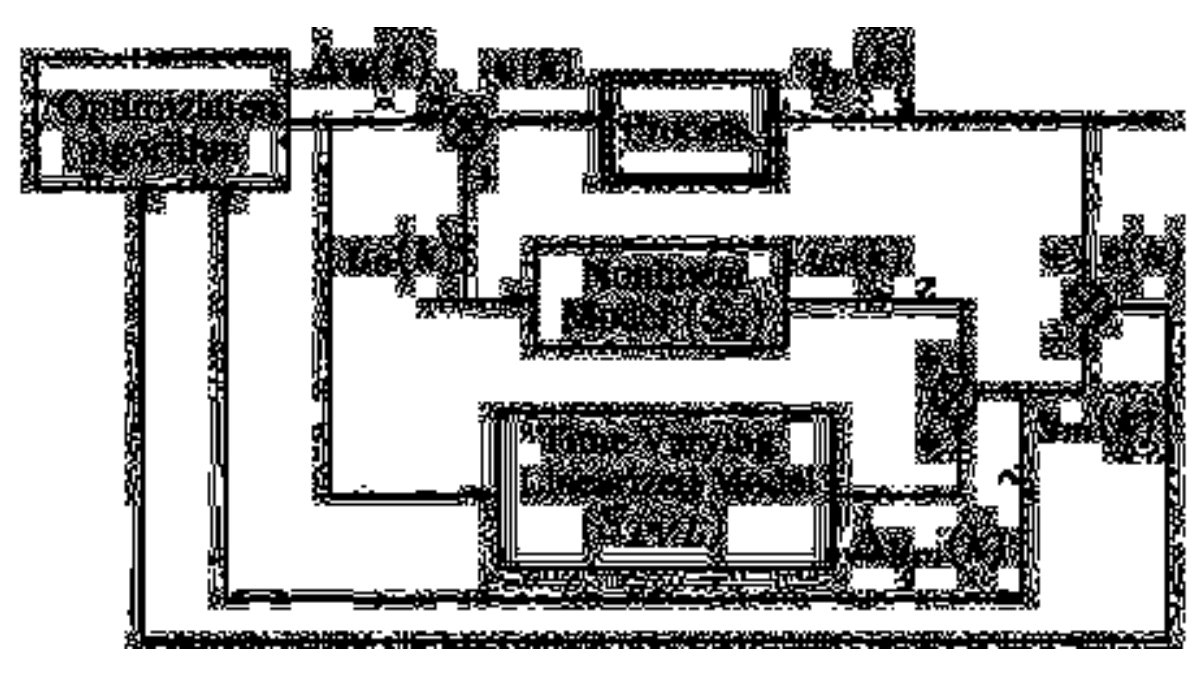

Figure 2: General linearized IMC-MPC structure (Dufour et al., 2003).

The control objective is then to find online the variation $\Delta d$ (hence $\Delta u$ ) of the variable $d$ (hence the manipulated variable $u$ ) about a well chosen trajectory $d_{0}$ (hence $u_{0}$ ) that improves at each sample time the online optimization result, based on the model response $\Delta y_{m}$. The final unconstrained penalized control problem that should be solved at each discrete time $k$ is:

$$
\min _{\Delta d} J_{t o t}(\Delta d)=\sum_{j=k+1}^{j=k+N_{p}} h_{2}\left(y_{r e f}(j), e(k), \Delta y_{m}(j), \Delta d(k)\right)
$$

with the iterative modified Levenberg Marquardt based algorithm:

$$
\Delta d^{l+1}(k)=\Delta d^{l}(k)-\left(\nabla^{2} J_{\text {tot }}^{l}(k)+\lambda I\right)^{-1} \nabla J_{\text {tot }}^{l}(k)
$$

where $\nabla J_{t o t}^{l}(k)$ and $\nabla^{2} J_{\text {tot }}^{l}(k)$ are the criteria gradient and criteria Hessian with respect to $\Delta d$ at the iteration $l$ at the time $k$. This algorithm has been implemented in the MPC@CB software ${ }^{3}$, which is used here.

\section{Simulation results and discussion}

\footnotetext{
${ }^{3}$ C University Claude Bernard Lyon 1 - EZUS. http://MPC-AT-CB.univ-lyon1.fr
} 
In order to control the sublimation front position, two closed loop control problems are presented here, assuming various availability of measurement used in the feedback loop:

- First, a trajectory tracking for a temperature (measured at the bottom of the sample) is used for inferential control of the sublimation front position.

- Secondly, the maximization of the constrained sublimation front position is used assuming availability of the online measure of the sublimation front position.

In these simulations, one is also interested in verifying the robustness of the controller with respect to uncertainty in a parameter used in the model namely the heat capacity of the frozen layer $c_{p 2}$.

\subsection{Simulation conditions}

The simulations have been performed under the following conditions:

- Sampling time $T e$ is $60 \mathrm{~s}$.

- In order to be used in the closed loop controller, the PDE model is approximated by a finite difference method, with 10 discretization points in the dried layer and 10 discretization points in the frozen layers.

- The values of the parameters of the model (9.-11.) are as reported in Table 1.

- The manipulated variable is subjected to the magnitude constraints in the following form:

$$
\left\{\begin{array}{l}
T_{\min } \leq u(t)=T_{l p}(t)=T_{u p}(t) \leq T_{\max }, \quad \forall t>0 \\
T_{\min }=220 \mathrm{~K} \\
T_{\max }=260 \mathrm{~K}
\end{array}\right.
$$

- In order to obtain a good quality product, the product temperature should never exceed its eutectic (or glass transition) temperature $T_{g}$. Indeed, if this temperature is exceeded, ice in the vial begins to liquefy and the concentrated phase temperature increases above $T_{g}$. Therefore the measured temperature at the bottom of the vial has to satisfy: 


$$
\left\{\begin{array}{l}
T_{2}(z=L, t) \leq T_{g}, \quad \forall t>0 \\
T_{g}=243 \mathrm{~K}
\end{array}\right.
$$

- In order to understand the influence of the uncertainty of the heat capacity value in the frozen layer $c_{p 2}$ used in the model, several conditions have been simulated assuming:

o the heat capacity of the frozen layer in the simulated process takes its nominal value, which is also used in the model.

○ the heat capacity of the frozen layer in the simulated process is either $24 \%$ or $50 \%$ bigger than the heat capacity of the frozen layer used in the model (the model is still assuming a nominal case).

The robustness of the controller with respect to this uncertainty is of major importance to validate the control approach. This aspect is discussed in the results.

\begin{tabular}{ccc}
\hline Parameter & Value & Unit \\
\hline$A$ & 6320.1517 & $\mathrm{~K}$ \\
\hline$B$ & 29.5578 & - \\
\hline$c_{p 1}$ & 2590 & $\mathrm{~J} / \mathrm{kg} / \mathrm{K}$ \\
\hline$c_{p 2}$ & 2500 (nominal case) & $\mathrm{J} / \mathrm{kg} / \mathrm{K}$ \\
\hline$c_{p g}$ & 1616.16 & $\mathrm{~J} / \mathrm{kg}$ \\
\hline$C_{s w}^{0}$ & & $\mathrm{~kg}$ water $/ \mathrm{kg}$ solid \\
\hline$F_{u p}$ & 0.6415 & - \\
\hline$h_{v}$ & 0.795 & $\mathrm{~W} / \mathrm{K} / \mathrm{m} 2$ \\
\hline
\end{tabular}




\begin{tabular}{|c|c|c|}
\hline$k_{1}$ & 0.003 & $\mathrm{~m}^{2} / \mathrm{s}$ \\
\hline$k_{1 e 1}$ & $2.596 * 10^{-4}$ & $\mathrm{~W} . \mathrm{m} / \mathrm{K} / \mathrm{N}$ \\
\hline$k_{1 e 2}$ & $3.9806^{*} 10^{-2}$ & $\mathrm{~W} / \mathrm{m} / \mathrm{K}$ \\
\hline$k_{2}$ & 2.1 & $\mathrm{~W} / \mathrm{m} / \mathrm{K}$ \\
\hline$k_{d}$ & $6.48 * 10^{-7}$ & $\mathrm{~s}^{-1}$ \\
\hline$L$ & 0.01 & $\mathrm{~m}$ \\
\hline$M_{w}$ & $18 * 10^{-3}$ & $\mathrm{~kg} / \mathrm{mol}$ \\
\hline$p_{0}$ & 5 & $\mathrm{~N} / \mathrm{m}^{2}$ \\
\hline$R_{i g}$ & 8.314 & N.m/K/mol \\
\hline$T^{O}$ & 233 & $\mathrm{~K}$ \\
\hline$\Delta H_{s}$ & $2840 * 10^{3}$ & $\mathrm{~J} / \mathrm{kg}$ \\
\hline$\Delta H v$ & $2687.4 * 10^{3}$ & $\mathrm{~J} / \mathrm{kg}$ \\
\hline$\rho_{1}$ & 60 & $\mathrm{~kg} / \mathrm{m}^{3}$ \\
\hline$\rho_{\text {le }}$ & 60 & $\mathrm{~kg} / \mathrm{m}^{3}$ \\
\hline$\rho_{2}$ & 1030 & $\mathrm{~kg} / \mathrm{m}^{3}$ \\
\hline$\sigma$ & $5.67 * 10^{-8}$ & $\mathrm{~W} / \mathrm{m}^{2} / \mathrm{K}^{4}$ \\
\hline
\end{tabular}

Table 1: Model parameter values.

\subsection{Criteria for results analysis}

In order to compare the various runs, several criteria are used: 
- the final time $t_{\text {final }}$, which is time when the sublimation front as reached the bottom of the vial at the end of the primary drying stage.

- the normalized root mean square (NRMS) error for the tracking (NRMSET) which represents, for a given run, the tracking error between the reference to track and the process output that has to track this reference:

$$
\text { NRMSET }=100 *\left(\sqrt{\frac{\sum_{k=1}^{k=N_{k}}\left(y_{r e f}(k)-y_{p}(k)\right)^{2}}{N_{k}}}\right) * \frac{1}{\left(\max _{k} y_{\text {ref }}(k)-\min _{k} y_{\text {ref }}(k)\right)}
$$

where $k$ is the time $t$ at the current sampled time and $N_{k}$ is the number of time samples of the considered run.

- the NRMS difference (NRMSD) which represents the variation of the same considered process output, obtained in the nominal run (run0) and in a run made under different numerical conditions (run1):

$$
N R M S D=100 *\left(\sqrt{\frac{\sum_{k=1}^{k=N_{k}}\left(y_{\text {run } 0}(k)-y_{\text {run } 1}(k)\right)^{2}}{N_{k}}}\right) * \frac{1}{\left(\max _{k} y_{\text {run } 0}(k)-\min _{k} y_{\text {run } 0}(k)\right)}
$$

where $k$ is the time $t$ at the current sampled time. Since run 0 and run 1 may have different duration, $N_{k}$ is the number of time samples of the fastest run. 
- the sublimation front mean velocity $(S F M V)$ during a run:

$$
S F M V=\sqrt{\frac{\sum_{k=1}^{k=N_{k}}\left(\frac{d H}{d t}(k)\right)^{2}}{N_{k}}}
$$

where $k$ is the time $t$ at the current sampled time and $N_{k}$ is the number of time samples of the considered run.

\subsection{Open loop control analysis}

Simulation results under open loop control, obtained with a constant value for $u$ during the run, are presented. These results allow evaluating the impact of uncertainty in the value of the heat capacity of the frozen layer $c_{p 2}$ over the position of the sublimation front and the temperature at the bottom of the vial. Figure 3 shows the sublimation front position (top) and the temperature at the bottom of the vial (bottom) for the same open loop control value (250.2 $\mathrm{K})$, but for 3 different uncertainties in the heat capacity of the frozen layer. The larger the value of this heat capacity, the higher the temperature, hence reducing the sublimation time. 

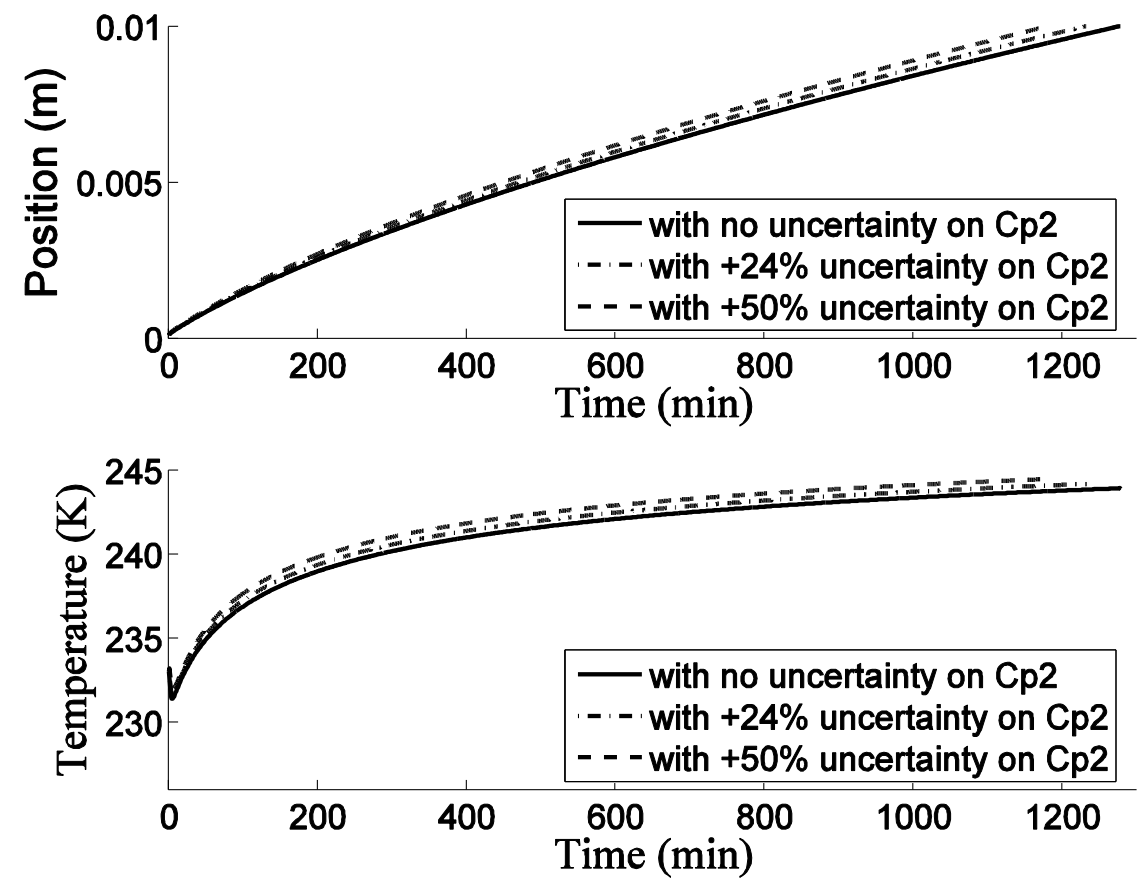

Figure 3: Open loop control case for various uncertainties in the heat capacity of the frozen layer: sublimation front position (top) and temperature at the bottom of the vial (bottom).

These results are summarized in Table 2: the variations of $t_{\text {final }}$ and the NRMSD range between 0 and $10 \%$. It tells us that if $c_{p 2}$ is not well known, the dynamics of the sublimation front position and temperature at the bottom of the vial may change, hence changing the duration of the primary drying stage. Therefore, it can be concluded that uncertainty in the specific heat has a strong impact over the outputs. In order to get a tight control of the sublimation front position at the end of the primary drying stage, a closed controller should therefore be used.

\begin{tabular}{ccc}
\hline Uncertainty in $c_{p 2}(\%)$ & NRMSD $(\%)$ & $t_{\text {final }}(\mathrm{min})$ \\
& & {$[\%$ variation with nominal case] } \\
\hline 0 (nominal case) & 0 & $1278[0]$ \\
\hline+24 & 2.9 & $1232[-3.6 \%]$ \\
\hline+50 & 6.4 & $1182[-7.5 \%]$
\end{tabular}


Table 2: Open loop control case for various uncertainties in the heat capacity of the frozen layer: Influence of the modeling error in $c_{p 2}$ on the comparison criteria.

\subsection{Inferential closed loop control of the sublimation time by temperature trajectory tracking}

In order to be able to control the sublimation front position in the case of uncertainty in heat capacity of the frozen layer, a first closed loop control approach is presented here: one assumes that the temperature at the bottom of the sample is measured (or estimated by a model-based sensor (Velardi et al., 2005, 2009)). It allows an inferential closed loop control of the key variable of interest: the sublimation front position. The optimization problem (13.) may be written here as the following trajectory tracking problem:

$$
\min _{u} \quad J(u)=\sum_{j=k+1}^{j=K+N_{p}}\left(y_{r e f}(j)-y_{p}(j)\right)^{2}
$$

where the manipulated variable $u(t)$ is the heating plate temperature $T_{u p}(t)$, the controlled variable $y_{p}(t)$ is the measured temperature $T_{2}(z=L, t)$, and the input constraints (23.) have to be satisfied. Here, the idea is to see how a prescribed reference behavior $y_{r e f}$ may be tracked online. This prescribed reference behavior may be tuned in two manners: (1) as a solution of an offline constrained model-based optimal problem (Dufour et al., 2004), in terms of drying time and final end-use properties of the product in the present case, or (2) based on practical knowledge to get an acceptable final product. Today, modeling of the end-use properties of the product based on the drying cycle does not yet exist. Therefore, it is assumed that the 
reference behavior $y_{r e f}$ is defined under some practical aspects: it is defined as a first rise of the controlled temperature with a constant ramp (to speed up the drying), then the temperature is kept at a constant value (to homogenize the space dependant dynamics). Finally, this behavior is repeated to increase the temperature to finish the drying. The linearization of (12.) is obtained after some trial and error (in terms of results obtained during the minimization of the cost function) about the behavior of $\left(S_{0}\right)$ given by $u_{0}=236.6 \mathrm{~K}$. The prediction horizon is 6 minutes. The optimization procedure runs until the end of the primary drying stage, i.e. when the position of the sublimation front in the process $H(t)$ reaches the length $L$.

Figure 4 represents the evolution of the manipulated boundary temperature (top) and the controlled temperature (bottom) during the drying time: the tracking is very accurate, in spite of the uncertainty introduced in the heat capacity of the frozen layer of the simulated process.

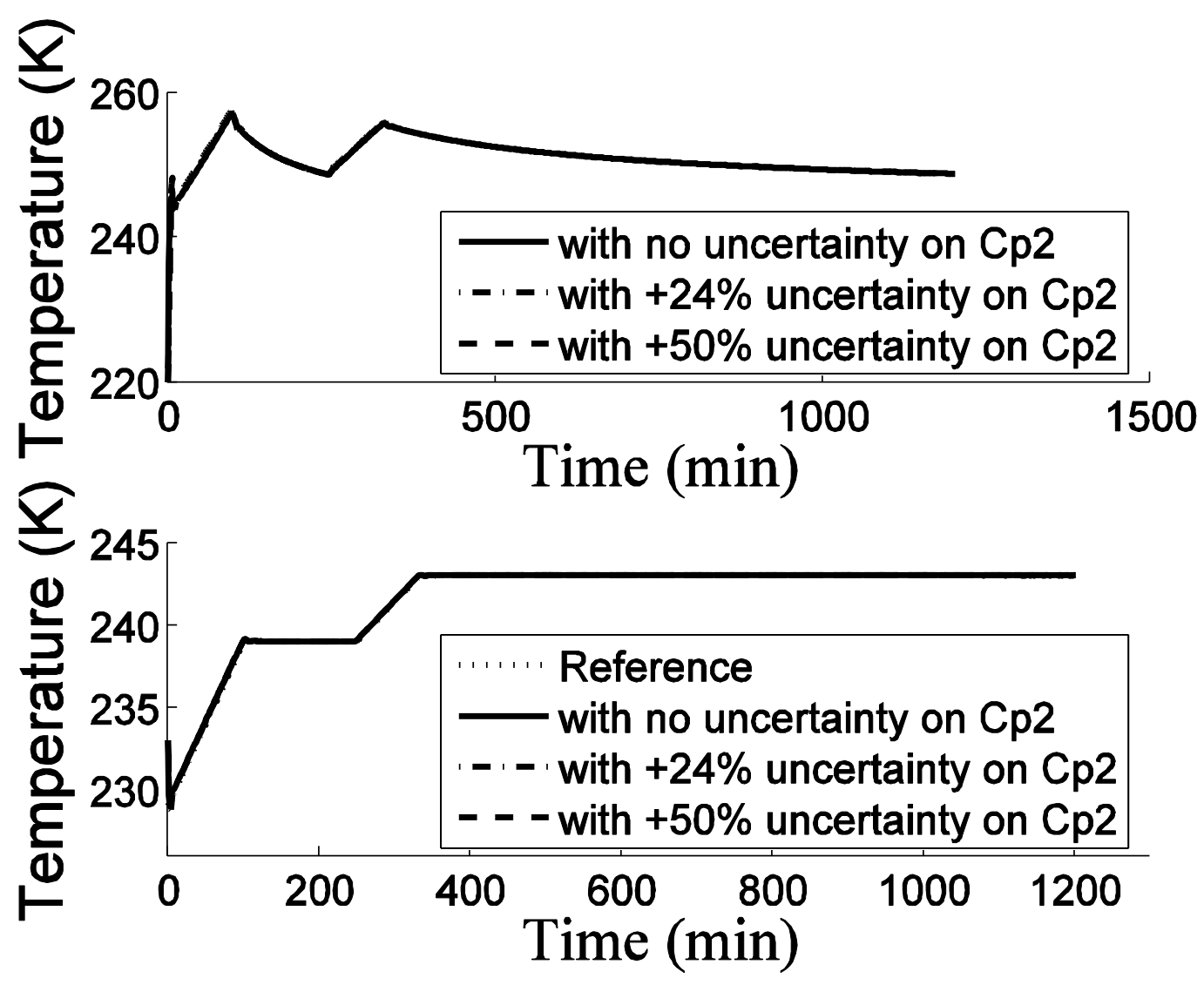


Figure 4: Inferential closed loop control case for various uncertainties in the heat capacity of the frozen layer: temperatures in the heating plate (top) and at the bottom of the vial (bottom).

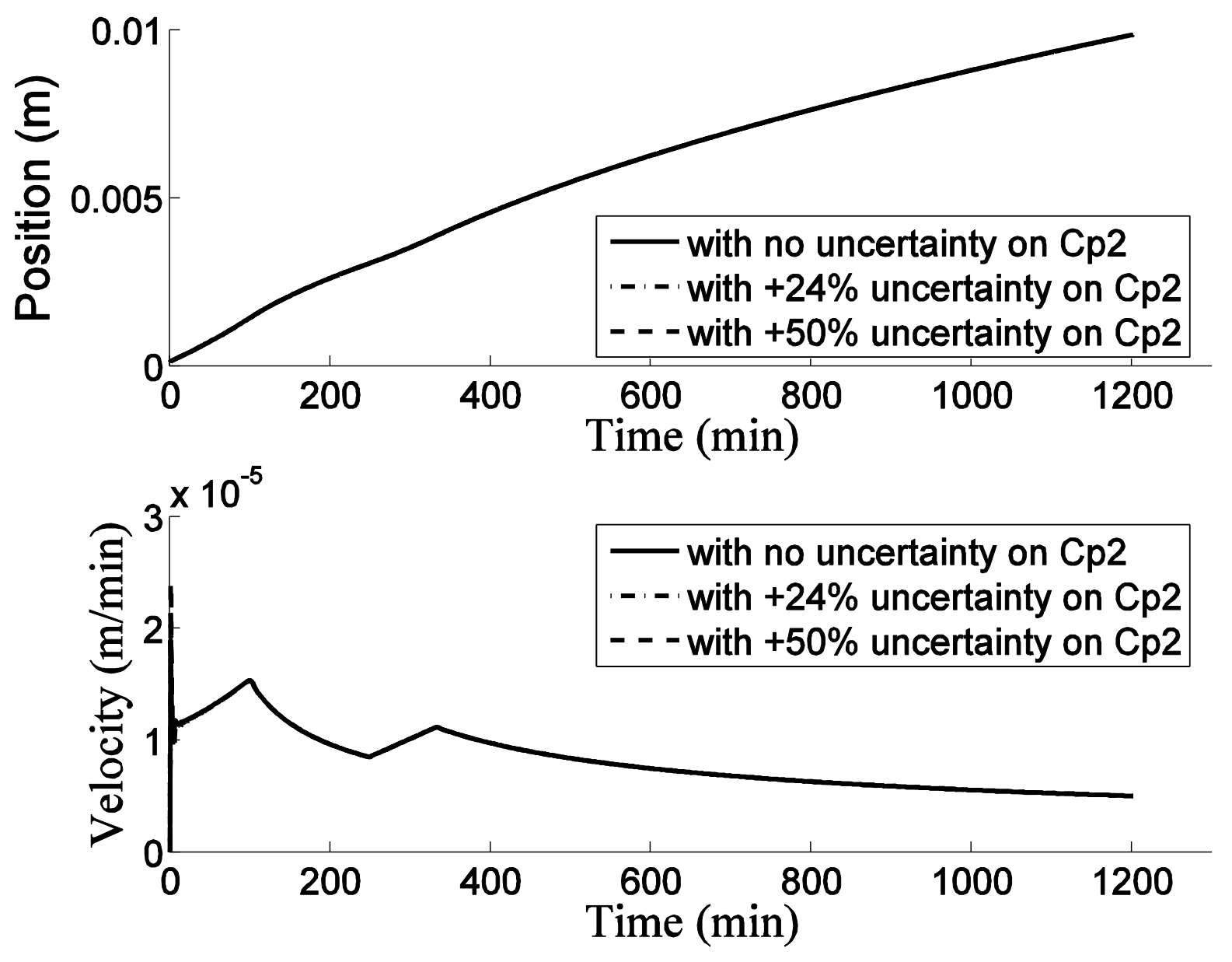

Figure 5: Inferential closed loop control case for various uncertainties in the heat capacity of the frozen layer: sublimation front position (top) and sublimation front velocity (bottom).

In the meantime, the inferential control of the sublimation front position is efficient (Figure 5) since it follows almost the same trajectory (top) in the 3 cases, hence decreasing the variation of the time at the end of the simulation. These results are summarized in Table 3: the variations of $\mathrm{t}_{\text {final, }}$ the NRMSD and the NRMSET are less than $1 \%$, and the sublimation front mean velocity (bottom of Figure 5) is almost similar in all cases. Using a closed loop 
controller instead of an open loop control policy allows decreasing these criterions by a factor 10, which allows a repeatability of the runs under parameter uncertainty. In that sense, the closed loop controller features a robust property, and allows an inferential control of the sublimation front position.

\begin{tabular}{|c|c|c|c|c|}
\hline $\begin{array}{l}\text { Uncertainty } \\
\text { in } c_{p 2}(\%)\end{array}$ & $\begin{array}{c}\text { NRMSET } \\
(\%)\end{array}$ & $\begin{array}{c}\text { NRMSD } \\
(\%)\end{array}$ & $\begin{array}{c}t_{\text {final }}(\mathrm{min}) \\
{[\% \text { variation with the }} \\
\text { nominal case] }\end{array}$ & $\begin{array}{l}\text { SFMV }(\mathrm{m} / \mathrm{min}) \\
{[\% \text { variation with the }} \\
\text { nominal case] }\end{array}$ \\
\hline 0 (nominal case) & 0.96 & 0 & 1201 & $8.50 * 10^{-6}$ \\
\hline+24 & 0.98 & 0.17 & $1201[-0.0 \%]$ & $8.50 * 10^{-6}[+0.01 \%]$ \\
\hline+50 & 1.03 & 0.33 & $1191[-0.8 \%]$ & $8.53 * 10^{-6}[+0.3 \%]$ \\
\hline
\end{tabular}

Table 3: Inferential closed loop control case for various uncertainties in the heat capacity of the frozen layer: Influence of the modeling error in $c_{p 2}$ on the comparison criteria.

\subsection{Minimization of the sublimation time under constraint}

In order to decrease the sublimation time, a second closed loop control approach is presented here based on the availability of the online measure of the sublimation front position (Velardi et al., 2005, 2009). It allows a direct control of the velocity of the sublimation front move. The optimal reduction of the drying time may be equivalent to define the optimal problem (13.) as the maximization of the velocity of the sublimation interface $\frac{d H(t)}{d t}$ : 


$$
\min _{u} J=\sum_{j=k+1}^{j=k+N_{p}} \frac{1}{\left\|\vartheta+\frac{d H}{d t}(j)\right\|^{2}}
$$

where $\vartheta$ is a small positive parameter introduced to avoid division by zero. The constraints for the manipulated variable (23.) and process output (24.) have to be satisfied, and limit the minimization of $t_{\text {final }}$. For the operating conditions, after trial and error, $u_{0}$ has been retuned to $239.22 \mathrm{~K}$. The prediction horizon is 20 minutes. The optimization procedure, based on the MPC@CB software, runs until the end of the primary drying stage, i.e. when the position of the sublimation front in the process $H(t)$ reaches the length $L$.

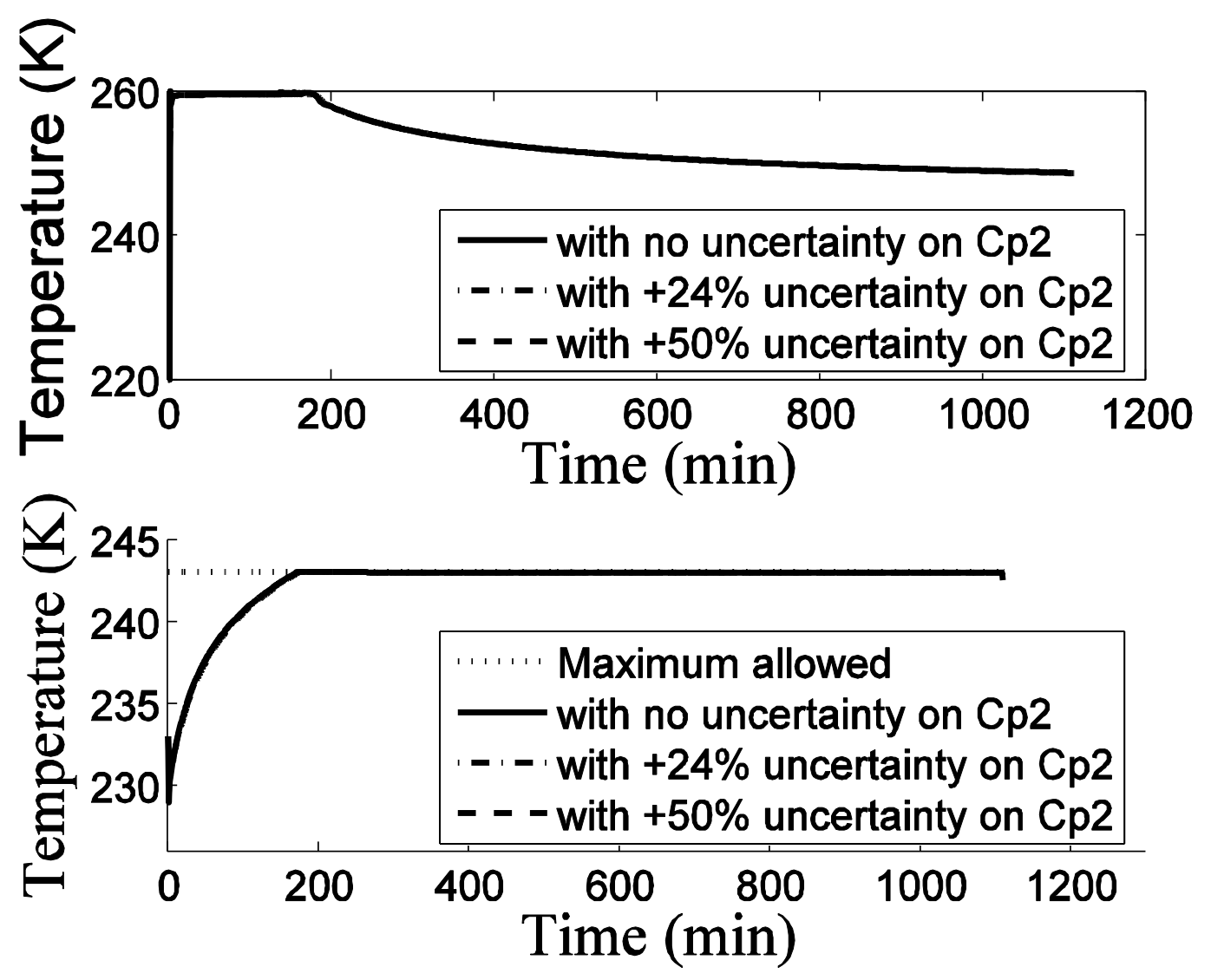

Figure 6: Constrained minimization of the sublimation time case for various uncertainties in the heat capacity of the frozen layer: temperatures in the heating plate (top) and at the bottom of the vial (bottom). 

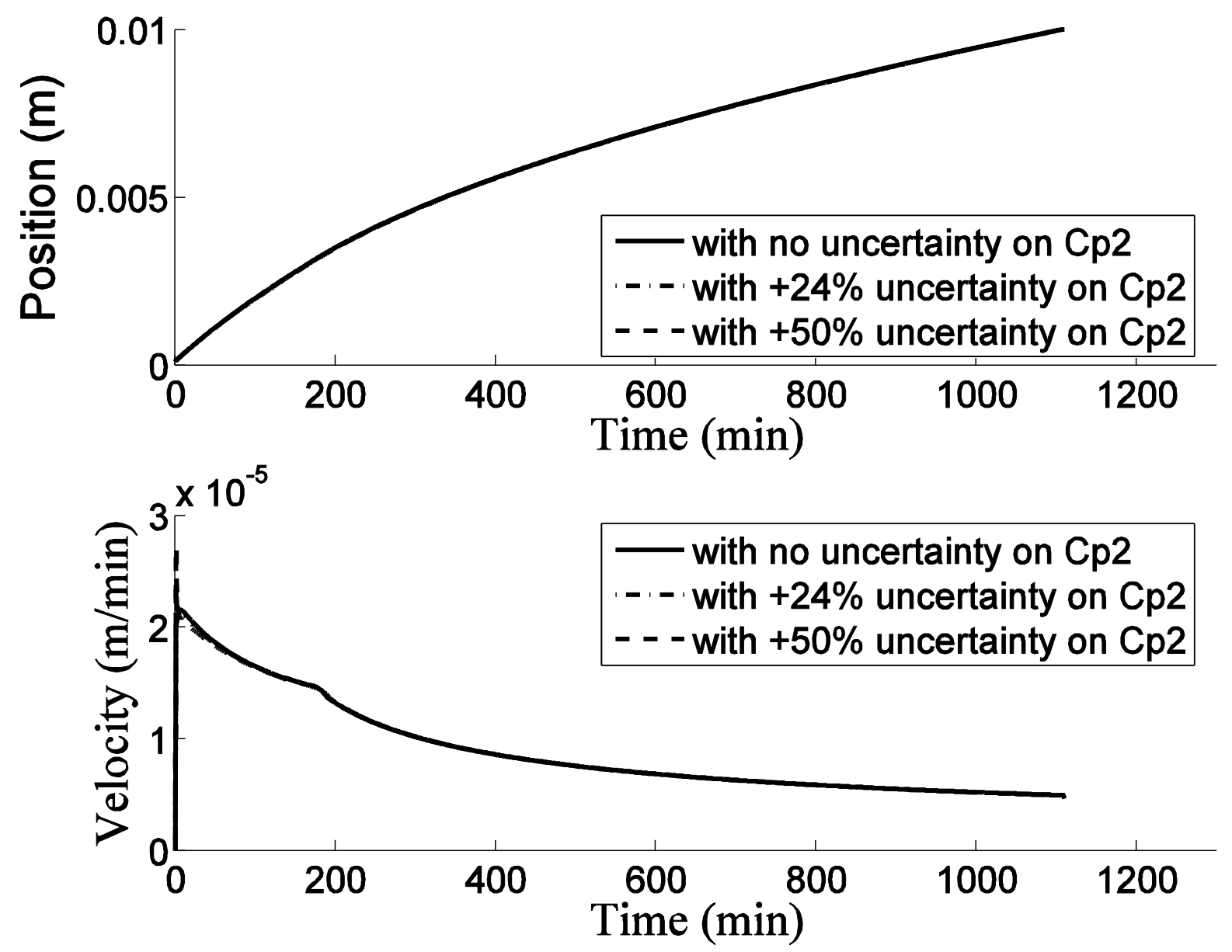

Figure 7: Constrained minimization of the sublimation time case for various uncertainties in the heat capacity of the frozen layer: sublimation front position (top) and sublimation front velocity (bottom).

Figure 6 represents the evolution of the manipulated boundary temperature (top) and the controlled temperature (bottom) during the drying time. The optimization results show consistent closed loop performance, in spite of the uncertainty introduced in the heat capacity of the frozen layer in the simulated process. Similarly, the optimization of the sublimation front velocity is efficient (bottom of Figure 7) and is almost similar in the 3 cases. Therefore, compared to the trajectory tracking problem, it allows decreasing the duration of the primary drying stage. Moreover, the output constraint (24.) is always satisfied and limits the 
sublimation front velocity when it becomes saturated (about 170 minutes). These results are summarized in Table 4: the variations of $\mathrm{t}_{\text {final }}$, the mean velocity of the sublimation front, the NRMSD for the temperature at the front are less than $0.5 \%$, which allows a consistent performance of the drying process under parameter uncertainty. Moreover, solving such constrained optimal control problem instead of a closed loop trajectory tracking problem allows decreasing the sublimation time up to $7 \%$. In that sense, this closed loop controller also features a robust property, and allows the minimization of the sublimation front position, while preserving the prescribed product properties.

\begin{tabular}{cccc}
\hline & & $t_{\text {final }}(\mathrm{min})$ & $S F M V(\mathrm{~m} / \mathrm{min})$ \\
Uncertainty & NRMSD & [\% variations: with the & [\% variations: with the \\
in $c_{p 2}(\%)$ & $(\%)$ & nominal case, and with the & nominal case, and with the \\
& & trajectory tracking problem $]$ & trajectory tracking problem] \\
\hline 0 (nominal case) & 0 & $1109[0 \%][-7.7 \%]$ & $9.88 * 10^{-6}[0 \%][+16.2 \%]$ \\
\hline+24 & 0.26 & $1110[+0.09 \%][-7.6 \%]$ & $9.86 * 10^{-6}[-0.2 \%][+16.0 \%]$ \\
\hline+50 & 0.50 & $1111[+0.18 \%][-6.7 \%]$ & $9.84 * 10^{-6}[-0.4 \%][+15.4 \%]$
\end{tabular}

Table 4: Constrained minimization of the sublimation time case for various uncertainties in the heat capacity of the frozen layer: Influence of the modeling error in $c_{p 2}$ on the comparison criteria.

\section{Conclusions}

This study tackled the model-based predictive control of the primary drying stage of the freeze drying process. The aim was to control online the sublimation front position (hence the 
drying time) in order to obtain dried products of highest quality at lower cost, in spite of a large parameter uncertainty in the model used in the controller. Due to the lack of online sensors, two closed loop control approaches were proposed to control the sublimation front position. First, a trajectory tracking of the measured temperature (at the bottom of the vial) allowed the inferential control of the unmeasured sublimation front position with an accuracy of $1 \%$, in spite of a large uncertainty of the value of the heat capacity in the frozen layer $(+50 \%)$. Secondly, a direct minimization of the sublimation time was shown, with the same accuracy. Compared to the first control approach, the second control approach allowed decreasing the sublimation time up to $7 \%$, while keeping the measured temperature inside a prescribed set (that allowed preserving final physical properties of the product). Compared to the open loop cases, the process performances could therefore be improved in spite of the approximations and the modeling errors introduced in the model based online optimizer: it allowed a repeatability of the runs even with parameter uncertainty.

\section{References}

Barresi AA, Velardi SA, Pisano R, Rasetto V, Vallan A, Galan M. In-line control of the lyophilization process A gentle pat approach using software sensors. International Journal of Refrigeration 2009; 32(5):1003-1014.

Besançon G. Non-linear observers and applications. Lecture Notes in Control and Information Science 363, Springer Verlag; 2007.

Boss EA, Filho RM, Vasco de Toledo, EC. Freeze drying process: Real time model and optimization. Chemical Engineering and Processing 2004; 43(12):1475-1485. 
Brülls M, Rasmuson A. Heat transfer in vial lyophilisation. International Journal of Pharmaceutics 2002;246(1-2):1-16.

Chouvenc P, Vessot S, Andrieu J, Vacus P. Optimization of a freeze-drying cycle: A new model for pressure rise analysis. Drying Technology 2004;22(7), 1577- 1601.

Christofides PD, El-Farra N, Li M, Mhaskar P. Model-based control of particulate processes. Chemical Engineering Science 2008;63:1156-1172.

Damak T. Procedure for asymptotic state and parameter estimation of non-linear distributed parameter bioreactors. Applied Mathematical Modeling 2007;31:1293-1307.

De Temmerman J, Dufour P, Nicolaï B, Ramon H. MPC as control strategy for pasta drying processes. Computers and Chemical Engineering 2009; 33(1):50-57.

Delgado AE, Sun DW. Heat and mass transfer models for predicting freezing processes - a review. Journal of Food Engineering 2001;47(3):157-174.

Diehl M, Bock HG, Schlöder JP, Findeisen R, Nagy Z, Allgöwer F. Real-time optimization and nonlinear model predictive control of processes governed by differential-algebraic equations. Journal of Process Control 2002;12:577-585.

Doyle III FJ. Non-linear inferential control for process applications. Journal of Process Control 1998; 8(5-6):339-353. 
Dubljevic S, Christofides PD. Predictive control of parabolic PDEs with boundary control actuation. Chemical Engineering Science 2006;61:6239-6248.

Dubljevic S, Mhaskar P, El-Farra NH, Christofides PD. Predictive control of transportreaction processes. Computers and Chemical Engineering 2005;29(11-12):2335-2345.

Duda JL, Malone MF, Notter RH, Vrentas JS. Analysis of two-dimensional diffusioncontrolled moving boundary problems. International Journal of Heat and Mass Transfer 1975; 18(7-8):901-910.

Dufour P. Control engineering in drying technology: Review and trends. Drying Technology 2006;24:889-904

Dufour P, Touré Y, Blanc D, Laurent P. On non-linear distributed parameter model predictive control strategy: On-line calculation time reduction and application to an experimental drying process. Computers and Chemical Engineering 2003; 27:1533-1542.

Dufour P, Touré Y, Michaud DJ, Dhurjati PS. A partial differential equation model predictive control strategy: Application to autoclave composite processing. Computers and Chemical Engineering 2004; 28(4), 545-556.

Fletcher R. Practical methods of optimization. New York: Wiley; 1987. 
Fissore D, Velardi SA, Barresi AA. In-line control of a freeze-drying process in vials. Drying Technology 2008;26 (6):685-694.

Hottot A, Peczalski R, Vessot S, Andrieu J. Freeze drying of pharmaceutical proteins in vials: Modelling of freezing and sublimation step. Drying Technology 2006a;24 (5):561-570.

Hottot A, Daoussi R, Andrieu J. Thermophysical properties of aqueous and frozen states of BSA/water/Tris systems International. Journal of Biological Macromolecules 2006b;38:225231.

Hottot A, Andrieu J, Shalaev E, Gatlin LA, Ricketts S. Experimental study and modeling of freeze-drying in syringe configuration. Part I: Freezing step Drying Technology 2009a;27(1): $40-48$.

Hottot A, Andrieu J, Hoang, V, Shalaev E, Gatlin LA, Ricketts S. Experimental study and modeling of freeze-drying in syringe configuration part II: Mass and heat transfer parameters and sublimation end-points. Drying Technology 2009b;27(1):49-58.

King CJ. Freeze-drying of foods. Cleveland, OH: CRC Press; 1971.

Li M, Christofides PD. Optimal control of diffusion-convection-reaction processes using reduced-order models. Computers and Chemical Engineering 2008;32:2123-2135.

Liapis AI, Litchfield RJ. Optimal control of a freeze dryer-I: theoretical development and quasi steady state analysis. Chemical Engineering Science 1979a;34:975-981. 
Liapis AI, Litchfield RJ. Numerical solution of moving boundary transport problems in finite media by orthogonal collocation. Computers and Chemical Engineering 1979b;3,:615-621.

Liapis AI, Bruttini R. A theory for the primary and secondary drying stages of the freeze drying of pharmaceutical crystalline and amorphous solutes; comparison between experimental data and theory. Separation Technology 1994;4:144-155.

Liapis AI, Pikal MJ, Bruttini R. Research and development needs and opportunities in freeze drying. Drying Technology 1996;14:1265-1300.

Litchfield RJ, Liapis AI. An adsorption-sublimation model for a freeze dryer. Chemical Engineering Science 1979;34(9):1085-1090.

Litchfield, R J, Liapis AI. Optimal control of a freeze Dryer-II: Dynamic analysis Chemical Engineering Science 1982;37(1):45-55.

Liu Y, Zhao Y, Feng X. Exergy analysis for a freeze-drying process. Applied Thermal Engineering 2008;28(7):675-690.

Lombraña JI, Díaz JM. Heat programming to improve efficiency in a batch freeze-drier. Chemical Engineering Journal 1987a;35(3):B23-B30.

Lombraña JI, Díaz JM. Coupled vacuum and heating power control for freeze-drying time reduction of solutions in phials. Vacuum 1987b;7(5-6):473-476. 
Lombraña JI, Villarán MC. The influence of pressure and temperature on freeze-drying in an adsorbent medium and establishment of drying strategies. Food Research International 1997; 30(3-4):213-222.

Mahmood M, Mhaskar P. Enhanced stability regions for nonlinear process systems using model predictive control. AIChE Journal 2008; 6:1487-1498.

Maidi A, Diaf M, Corriou JP. Boundary geometric control of a counter-current heat exchanger. Journal of Process Control 2009;19(2):297-313.

Mascarenhas WJ, Akay HU, Pikal MJ. A computational model for finite element analysis of the freeze-drying process. Computer Methods in Applied Mechanics and Engineering $1997 ; 148(1-2): 105-124$.

Meo III D, Friedly JC. An experimental study of the optimal feedback control of a freeze dryer. Journal of Food Science 1973;38(5):826-830.

Nakagawa K, Hottot A, Vessot S, Andrieu J. Modeling of freezing step during freeze-drying of drugs in vials. AIChE Journal 2007;53(5):1362-1372.

Padhiyar N, Bhartiya S. Profile control in distributed parameter systems using lexicographic optimization based MPC. Journal of Process Control 2009;19(1):100-109. 
Schäfer A, Kühl P, Diehl M, Schlöder J, Bock HG. Fast reduced multiple shooting methods for nonlinear model predictive control. Chemical Engineering and Processing 2007; 46:12001214.

Sheehan P, Liapis AI. Modeling of the primary and secondary drying stages of the freezedrying of pharmaceutical products in containers: numerical results obtained from the solution of a dynamic and spatially multi-dimensional lyophilization model for different operational policies. Biotechnology and Bioengineering 1998;60 712-728.

Tang X, Nail SL, Pikal MJ. Freeze-drying process design by manometric temperature measurement: Design of a smart freeze-dryer. Pharmaceutical Research 2005;22(4):685-700.

Velardi SA. Freeze-drying: Modelling monitoring and control. PhD Thesis of the Politecnico di Torino Italy, 2004.

Velardi SA, Barresi AA. Development of simplified models for the freeze-drying process and investigation of the optimal operating conditions. Chemical Engineering Research and Design 2008;86(1):9-22.

Velardi SA, Hammouri H, Barresi AA. A non-linear observer for real-time monitoring and control of the freeze-drying process of pharmaceuticals in vial, in Proceedings of the Joint Conference of AFSIA and Drying Working group of EFCE, Paris, France, 28-29, 2005. 
Pikal MJ. Freeze drying. Encyclopedia of Pharmaceutical Technology Third Edition. Taylor \& Francis; 2006.

Qin SJ, Badgwell TA. A survey of industrial model predictive control technology. Control Engineering Practice 2003;11(7):733-764.

Ravindran SS. Optimal boundary feedback flow stabilization by model reduction. Computer Methods in Applied Mechanics and Engineering 2007; 196:2555-2569.

Sadikoglu H, Liapis AI. Mathematical modelling of the primary and secondary drying stages of bulk solution freeze-drying in trays: Parameter estimation and model discrimination by comparison of theoretical results with experimental data. Drying Technology 1997;15:791810.

Sadikoglu H, Liapis AI, Crosser OK. Optimal control of the primary and secondary drying stage of bulk solution freeze drying in trays. Drying Technology 1998;16:399-431

Sadikoglu H, Ozdemir M, Seker M. Optimal control of the primary drying stage of freeze drying of solutions in vials using variational calculus. Drying Technology 2003;21:13071331.

Sane SU, Hsu CC. Mathematical model for a large-scale freeze-drying process: A tool for efficient process development and routine production, in Proceedings of the 16th International Drying Symposium, Hyderabad, India, vol B, 680-688, 2008. 
Velardi SA, Hammouri H, Barresi AA. In-line monitoring of the primary drying phase of the freeze-drying process in vial by means of a Kalman filter based observer. Chemical Engineering Research and Design 2009; 87(10):1409-1419.

Zavala VM, Biegler LT. Optimization-based strategies for the operation of low-density polyethylene tubular reactors: nonlinear model predictive control. Computers and Chemical Engineering 2009, 33(10): 1735-1746.

\section{Nomenclature for the model}

\begin{tabular}{ll}
\hline A & Antoine's constant $(\mathrm{K})$ \\
\hline B & Antoine's constant (-) \\
\hline$c_{p 1}$ & heat capacity of the dried layer $(\mathrm{J} / \mathrm{kg} / \mathrm{K})$ \\
\hline$c_{p 2}$ & heat capacity of the frozen layer $(\mathrm{J} / \mathrm{kg} / \mathrm{K})$ \\
\hline$c_{p g}$ & specific heat capacity at constant pressure $(\mathrm{J} / \mathrm{kg})$ \\
\hline$C_{s w}$ & concentration of bound water $(\mathrm{kg}$ water $/ \mathrm{kg}$ solid $)$ \\
\hline$\tilde{C}_{S w}$ & concentration of bound water in the new coordinates $(\mathrm{kg}$ water $/ \mathrm{kg}$ solid $)$ \\
\hline$C_{S w}^{0}$ & initial concentration of bound water at $\mathrm{t}=0$ (kg water $/ \mathrm{kg}$ solid $)$ \\
\hline$F_{u p}$ & view factor for radiative heat transfer $(-)$ \\
\hline$H$ & sublimation front position (m) \\
\hline$h_{v}$ & heat transfer coefficient (W/K/m $\left.{ }^{2}\right)$ \\
\hline
\end{tabular}




\begin{tabular}{|c|c|}
\hline$\overline{k_{1}}$ & bulk diffusivity constant $\left(\mathrm{m}^{2} / \mathrm{s}\right)$ \\
\hline$k_{1 e}$ & thermal conductivity in the dried layer $(\mathrm{W} / \mathrm{m} / \mathrm{K})$ \\
\hline$\overline{k_{l e l}}$ & thermal conductivity coefficient in the dried layer $(\mathrm{W} . \mathrm{m} / \mathrm{K} / \mathrm{N})$ \\
\hline$\overline{k_{l e 2}}$ & thermal conductivity coefficient in the dried layer $(\mathrm{W} / \mathrm{m} / \mathrm{K})$ \\
\hline$\overline{k_{2}}$ & thermal conductivity in the frozen layer $(\mathrm{W} / \mathrm{m} / \mathrm{K})$ \\
\hline$\overline{k_{d}}$ & desorption rate constant of bound water $\left(\mathrm{s}^{-1}\right)$ \\
\hline$\overline{\mathrm{L}}$ & length of sample product in vial (m) \\
\hline$\overline{M_{w}}$ & molecular weight of water vapor $(\mathrm{kg} / \mathrm{mol})$ \\
\hline$\overline{N_{w}}$ & mass flux of the water vapor $\left(\mathrm{N} / \mathrm{m}^{2} / \mathrm{s}\right)$ \\
\hline$p_{0}$ & partial pressure of water vapor at $\mathrm{z}=0\left(\mathrm{~N} / \mathrm{m}^{2}\right)$ \\
\hline$p_{H}$ & partial pressure of water vapor at $\mathrm{z}=\mathrm{H}\left(\mathrm{N} / \mathrm{m}^{2}\right)$ \\
\hline$p_{H \infty}$ & partial pressure of water vapor coefficient $\left(\mathrm{N} / \mathrm{m}^{2}\right)$ \\
\hline$q_{1}$ & heat flux at the top of the dried layer at $\mathrm{z}=0\left(\mathrm{~W} / \mathrm{m}^{2}\right)$ \\
\hline$\overline{q_{2}}$ & heat flux at the bottom of the frozen layer at $\mathrm{z}=\mathrm{L}\left(\mathrm{W} / \mathrm{m}^{2}\right)$ \\
\hline$\overline{R_{i g}}$ & ideal gas constant $(\mathrm{N} . \mathrm{m} / \mathrm{K} / \mathrm{mol})$ \\
\hline$\overline{S F M V}$ & sublimation front mean velocity $(\mathrm{m} / \mathrm{min})$ \\
\hline $\mathrm{t}$ & time $(\mathrm{s})$ \\
\hline$\overline{t_{\text {final }}}$ & final time at the end of the simulation (s) \\
\hline$\overline{T^{0}}$ & initial product temperature at $\mathrm{t}=0(\mathrm{~K})$ \\
\hline$\overline{T_{l p}}$ & temperature of lower heating plate $(\mathrm{K})$ \\
\hline$\overline{T_{u p}}$ & temperature of the upper heating plate $(\mathrm{K})$ \\
\hline$\overline{T_{1}}$ & temperature in the dried layer $(\mathrm{K})$ \\
\hline
\end{tabular}




\begin{tabular}{ll}
\hline$\tilde{T}_{1}$ & temperature in the dried layer in the new coordinates $(\mathrm{K})$ \\
\hline$T_{2}$ & temperature in the frozen layer $(\mathrm{K})$ \\
\hline$\tilde{T}_{2}$ & temperature in the dried layer in the new coordinates $(\mathrm{K})$ \\
\hline$T_{g}$ & glass transition temperature $(\mathrm{K})$ \\
\hline$T_{\text {min }}$ & minimum temperature allowed in the chamber $(\mathrm{K})$ \\
\hline$T_{m a x}$ & maximum temperature allowed in the chamber $(\mathrm{K})$ \\
\hline $\mathrm{Z}$ & space variable (m) \\
\hline$\Delta H_{s}$ & enthalpy of sublimation of ice $(\mathrm{J} / \mathrm{kg})$ \\
\hline$\Delta H_{v}$ & enthalpy of vaporization of bound water $(\mathrm{J} / \mathrm{kg})$ \\
\hline$\rho_{1}$ & density in the dried layer $\left(\mathrm{kg} / \mathrm{m}^{3}\right)$ \\
\hline$\rho_{l e}$ & effective density in the dried layer $\left(\mathrm{kg} / \mathrm{m}^{3}\right)$ \\
\hline$\rho_{2}$ & density in the frozen layer $\left(\mathrm{kg} / \mathrm{m}^{3}\right)$ \\
\hline$\sigma$ & Stefan Boltzman constant $\left(\mathrm{W} / \mathrm{K}^{4} / \mathrm{m}^{2}\right)$ \\
\hline$\xi_{1}$ & normalized space variable in the dried layer $(-)$ \\
\hline
\end{tabular}

\section{Nomenclature for the control approach}

\begin{tabular}{ll}
\hline$d$ & Unconstrained manipulated variable \\
\hline$d_{0}$ & Unconstrained manipulated variable for $S_{0}$ \\
\hline$\Delta d$ & Unconstrained manipulated variable for $S_{T V L}$ \\
\hline$e$ & Output error \\
\hline
\end{tabular}




\begin{tabular}{|c|c|}
\hline$F_{b}, F_{d}$ & Nonlinear operators \\
\hline$g_{i}$ & Output constraints \\
\hline$h_{1}, h_{2}$ & Functions \\
\hline$j$ & Discrete time index in the future \\
\hline$k$ & Actual discrete time index \\
\hline$J$ & Cost function \\
\hline$J_{\text {ext }}$ & Exterior penalty function \\
\hline$J_{t o t}$ & Total penalized cost function \\
\hline$l$ & Iteration index in the algorithm \\
\hline$n$ & Number of output constraints \\
\hline$N_{k}$ & Number of time samples considered \\
\hline$N_{p}$ & Prediction horizon (-) \\
\hline NRMSET & Normalized root mean square error for tracking $(\%)$ \\
\hline NRMSD & Normalized root mean square difference $(\%)$ \\
\hline$S_{N L}$ & Nonlinear model \\
\hline$S_{0}$ & Nonlinear model computed off-line \\
\hline$S_{T V L}$ & Time-varying linearized model computed on-line \\
\hline$t$ & Time $(s)$ \\
\hline $\mathrm{Te}$ & Sampling time (s) \\
\hline$u$ & Manipulated variable \\
\hline$\Delta u_{\min }$ & Minimum variation allowed for the manipulated variable during one sample time \\
\hline$\Delta u_{\max }$ & Maximum variation allowed for the manipulated variable during one sample time \\
\hline
\end{tabular}




\begin{tabular}{|c|c|}
\hline$u_{\min }$ & Minimum magnitude allowed for the manipulated variable \\
\hline$u_{\max }$ & Maximum magnitude allowed for the manipulated variable \\
\hline$u_{0}$ & Manipulated variable for $S_{0}$ \\
\hline$\Delta u$ & Manipulated variable for $S_{T V L}$ \\
\hline$w_{i}$ & Penalty weight \\
\hline$x_{m}$ & Model state \\
\hline$x_{m}^{0}$ & Initial condition for the model state at $\mathrm{t}=0$ \\
\hline$\Delta x_{m}$ & Model state for $S_{T V L}$ \\
\hline$y_{m}$ & Model output \\
\hline$y_{0}$ & Model output for $S_{0}$ \\
\hline$\Delta y_{m}$ & Model output for $S_{T V L}$ \\
\hline$y_{p}$ & Process output \\
\hline$y_{\text {ref }}$ & Reference behavior \\
\hline$\nabla$. & Gradient operator \\
\hline$\nabla^{2}$ & Hessian operator \\
\hline$\lambda$ & Control algorithm parameter \\
\hline$\partial \Omega$ & Boundary of the spatial domain \\
\hline$\Omega$ & Spatial domain \\
\hline$\zeta$ & Space variable $(\mathrm{m})$ \\
\hline
\end{tabular}

